Aus dem Forschungsinstitut für die Biologie landwirtschaftlicher Nutztiere (FBN), Dummerstorf ${ }^{1}$ und der Landschaftspflege GmbH, Lenzen ${ }^{2}$

DELIA MICKLICH, HEIDE-DÖRTE MATTHES, MARGITTA HARTUNG ${ }^{1}$ und HORST MÖHRING ${ }^{2}$

\title{
Mast-, Schlachtleistung und Fleischqualität verschiedener Schweinerassen bei Stall- und Freilandhaltung (Kurzmitteilung)
}

\begin{abstract}
Summary
Title of the paper: Fattening, slaughter performance and meat quality of different pig breeds in indoor or outdoor housing (short communication)

Relationships between outdoor housing and some meat quality parameters were investigated. Studies were carried out with the pig breeds Schwerfurter Fleischrasse, Deutsches Sattelschwein und Duroc. Fattening, slaughter performances and meat quality were compared by indoor or outdoor housing. Between different breeds in most parameters there were significant differences. Regarding the influence of outdoor housing with grazing the study showed improve results by meat quality parameters only in meat colour and water keeping.
\end{abstract}

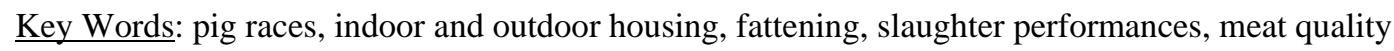

\section{Zusammenfassung}

Es wurde der Einfluss von Stall- und Freilandhaltung auf einige Merkmale der Fleischqualität beim Schwein untersucht. Als Tiermaterial konnten die Rassen Schwerfurter Fleischrasse, Deutsches Sattelschwein und Duroc einbezogen werden. Verglichen wurden die Mast- und Schlachtleistungen sowie Merkmale der Fleischqualität. Bei den meisten Merkmalen bestanden Unterschiede zwischen den einzelnen Rassen. Bezüglich der Fleischqualität zeigten sich nur bei der Fleischfarbe und dem Wasserhaltevermögen bessere Ergebnisse durch den Einfluss der Freilandhaltung.

Schlüsselwörter: Schweinerassen, Stallhaltung, Freilandhaltung, Mastleistung, Schlachtleistung, Fleischqualität

1. Einleitung

Eine Nachfrage nach Erzeugnissen aus einer naturnahen Produktion, die sich nicht immer berechtigt mit Gesichtspunkten der Qualitätsverbesserung verbinden, führen zu mehr tierfreundlichen Haltungsformen. Das schliesst Ställe mit ausgedehnten Bewegungsflächen ebenso ein, wie die Haltung von Zuchttieren oder Mastschweinen auf Weideflächen. Noch immer wird dann der grösste Erlös erzielt, wenn Schweine bei hohen Tageszunahmen und bei günstigen Futterkosten einen hohen Magerfleischanteil erreichen. Da zur Zeit weder subjektive noch objektive Qualitätsmerkmale des Produktes „Schweinefleisch“ bei der Bezahlung eine Rolle spielen, bleiben für Schweine aus der Freilandhaltung überwiegend ethische Gesichtspunkte, welche die Art und Weise gesundheitsfördernder Haltung bei der Preisbildung berücksichtigen. Nach den bisherigen Marktentwicklungen, haben diese alternativen Wege noch den Status von „Nischenprogrammen“. Die Frage ist, ob Schweine, die unter Einbeziehung der Freilandhaltung gemästet werden, sich bezüglich der Fleischqualität, von Tieren aus herkömmlichen Haltungsformen unterscheiden. Nachfolgend soll über Ergebnisse des 
Einflusses der Weidehaltung bei Tieren aus unterschiedlichen Rassen auf die Fleischqualität, am Beispiel ausgewählter Merkmale, berichtet werden.

\section{$2 . \quad$ Material und Methode}

Je drei Versuchsgruppen aus Börgen der Rassen Schwerfurter Fleischrasse (SF), Deutsches Sattelschwein (DS) und Duroc (DU) wurden sowohl in Weide- bzw. Stallhaltung 180 (175 - 186) Tage gemästet. Den Freilandgruppen standen für jeweils 10 Tiere und Rasse ein ha Weidefläche mittlerer Grünlandqualität im Zeitraum Juni bis November zur Verfügung. Die drei im Stall gemästeten Gruppen mit 10 Tieren je Rasse wurden mit jeweils 5 Tieren je Bucht auf Stroheinstreu gehalten. Sowohl die Freiland als auch die Stallgruppen erhielten je Tag eine Kraftfuttergabe von 0,5 kg je Tier (13 MJ ME, $17 \%$ PR, 0,9\% Lysin). Während die Weidegruppen das Kraftfutter einmal am Tag in Einzelfressständen bei ad libitum Grasaufnahme auf der Weide erhielten, wurde den Stalltieren einmal täglich Grünschnittfutter ad libitum gereicht. Ab dem 140. Tag betrug die tägliche Kraftfuttergabe für alle Versuchsgruppen 1,0 kg.

Die Schlachtung der Tiere erfolgte überwiegend nach 180 Masttagen (Tab. 1). Für die DS und DU Gruppen im Schlachthof Perleberg für die SF Gruppen im Schlachthaus des FBN Dummerstorf. Schlachtung und Zerlegung erfolgten nach den Richtlinien für die „Stationsprüfung auf Mastleistung, Schlachtkörperwert und Fleischbeschaffenheit beim Schwein“ (ZDS, 1997). An letzgenanntem Standort erfolgte für sämtliche Gruppen die Ermittlung der Fleischqualitätsparameter. Für die DS Stallgruppen konnten die Merkmale intramuskuläres Fett, L-Wert und Dripverlust nicht festgestellt werden.

Die ermittelten Daten sind mit Hilfe des Statistical-Analysis-Systems (SAS, 1990) bearbeitet worden. Die Prüfung der Einflüsse von Rasse und Stall erfolgte über eine einfache Varianzanalyse. Die Ergebnisse wurden als Mittelwerte für jede Rasse in jeder Haltungsform zusammen gefasst. Mittelwertdifferenzen wurden mittels t-Test auf Signifikanz geprüft, wobei eine Irrtumswahrscheinlichkeit von $\mathrm{P}<0,001 ; 0,01$ bzw. 0,05 angegeben wird.

\section{3. $\quad$ Ergebnisse}

Die Tabelle 1 zeigt die Ergebnisse der Mastleistungen der drei Rassen bei unterschiedlichen Haltungsformen.

Tabelle 1

Mastleistungen der Versuchsgruppen (Fattening parameters)

\begin{tabular}{|c|c|c|c|c|c|c|c|c|}
\hline \multirow[t]{2}{*}{ Rasse } & \multirow[t]{2}{*}{$\mathrm{n}$} & \multirow[t]{2}{*}{ Haltung } & \multicolumn{2}{|c|}{ Mastbeginn (kg) } & \multicolumn{2}{|c|}{ Mastende (kg) } & \multirow[t]{2}{*}{ Tage } & \multirow[t]{2}{*}{ tgl Zun. (g) } \\
\hline & & & & $\operatorname{lter}(c$ & & & & \\
\hline \multirow[t]{2}{*}{ SF } & 10 & Stall & 40,2 & 98 & 110,9 & B & 180 & 393 \\
\hline & 11 & Freiland & 41,8 & 100 & 111,7 & & 180 & 388 \\
\hline \multirow[t]{2}{*}{ DS } & 10 & Stall & 42,8 & 100 & 114,0 & A & 186 & 383 \\
\hline & 9 & Freiland & 43,9 & 100 & 112,6 & & 186 & 369 \\
\hline \multirow[t]{2}{*}{ DU } & 10 & Stall & 39,5 & 98 & 128,8 & $\mathrm{Aa}$ & 175 & 510 \\
\hline & 9 & Freiland & 41,7 & 98 & 109,6 & $\mathrm{~b}$ & 180 & 377 \\
\hline
\end{tabular}

unterschiedlich kleine Buchstaben = Signifikanz zwischen den Haltungsformen, innerhalb einer Rasse

unterschiedlich große Buchstaben = Signifikanz zwischen den Rassen, innerhalb der Haltungsform

Die DU Stallgruppe erzielte sowohl bei der Stallhaltung gegenüber der Freilandhaltung gleicher Rasse signifikant höhere Tageszunahmen als auch gegenüber den anderen Versuchsgruppen, die ein annährend gleiches relativ niedriges Niveau erreichten. 
Diese DU Stallgruppe hatte die geringsten Einstallungsgewichte bei geringster Mastdauer und den höchsten Ausstallungsgewichten.

Die Tabelle 2 zeigt die Ausschlachtungsergebnisse der drei Rassen bei unterschiedlichen Haltungsformen.

Tabelle 2

Schlachtgewichte und Muskelfleischanteil (Slaughter weights and lean meat)

\begin{tabular}{|c|c|c|c|c|c|c|c|c|}
\hline$\overline{\text { Rasse }}$ & $\mathrm{n}$ & $\begin{array}{l}\text { Stall } \\
\mathrm{LSM} \pm \mathrm{SD}\end{array}$ & & $\begin{array}{l}\text { Weide } \\
\mathrm{LSM} \pm \mathrm{SD}\end{array}$ & & $\begin{array}{l}\text { Einflussf } \\
\text { Haltung }\end{array}$ & $\begin{array}{l}\text { ktoren } \\
\text { Rasse } \\
\text { : Stall }\end{array}$ & Weide \\
\hline & & Mastendgewicht (kg) & & & & & & \\
\hline SF & 21 & $110,9 \pm 7,5$ & & $111,7 \pm 7,1$ & & n.s. & : B & - \\
\hline DS & 19 & $114,0 \pm 6,6$ & & $112,6 \pm 5,2$ & & n.s. & A & - \\
\hline DU & 19 & $\begin{array}{l}128,8 \pm 7,0 \\
\text { Schlachtgewicht (kg) }\end{array}$ & a & $109,6 \pm 7,3$ & $\mathrm{~b}$ & $* * *$ & A & - \\
\hline SF & 21 & $86,8 \pm 6,7$ & & $91,0 \pm 6,4$ & & n.s. & : B & - \\
\hline DS & 19 & $92,4 \pm 5,0$ & & $87,9 \pm 5,6$ & & n.s. & A & - \\
\hline DU & 19 & $\begin{array}{l}103,3 \pm 8,8 \\
\text { MFA (\%) }\end{array}$ & $\mathrm{a}$ & $83,9 \pm 8,4$ & $\mathrm{~b}$ & $* *$ & A & - \\
\hline SF & 21 & $51,9 \pm 3,2$ & & $48,9 \pm 3,0$ & & n.s. & A & $\mathrm{B}$ \\
\hline DS & 19 & $43,3 \pm 1,9$ & $\mathrm{~b}$ & $45,9 \pm 1,8$ & $\mathrm{a}$ & $*$ & $\mathrm{~B}$ & $\mathrm{C}$ \\
\hline Du & 19 & $52,6 \pm 2,1$ & & $53,4 \pm 1,8$ & & n.s. & A & A \\
\hline
\end{tabular}

unterschiedlich kleine Buchstaben = Signifikanz zwischen den Haltungsformen, innerhalb einer Rasse; *P $\leq 0,05, * * \mathrm{P} \leq 0,01,{ }^{* * *} \mathrm{P} \leq 0,001$ unterschiedlich große Buchstaben = Signifikanz zwischen den Rassen, innerhalb der Haltungsform, ${ }^{*} \mathrm{P} \leq 0,05$

Das Schlachtgewicht liegt bei DU signifikant höher als bei der Stallgruppe und den übrigen Gruppen, unabhängig von Rasse oder Haltungsform. Die Schlachtausbeute schwankte von 76,6\% bei der DU Weidegruppe bis 82,0\% bei der SF Weidegruppe. Zwischen den Stall- und Weidegruppen ergaben sich keine signifikanten Unterschiede. Hinsichtlich des Muskelfleischanteils ergab sich nur bei DS ein signifikant besseres Ergebnis der Weidegruppe, jedoch erreichten die Tiere dieser Rasse in beiden Haltungsformen erwartungsgemäß den geringsten, die Rasse DU bei beiden Haltungsformen den größten Muskelfleischanteil innerhalb des Rassenvergleiches.

Die Tabelle 3 zeigt den Ergebnisvergleich der Fleischqualitätsmerkmale.

Die Tiere der Rasse DU zeigten den höchsten intramuskulären Fettgehalt, wobei sich zwischen den beiden Haltungsformen keine Unterschiede ergaben. Das trifft, wenn auch mit niedrigerem intramuskulären Fettgehalt für die anderen Rassen zu. Erwartungsgemäß wiesen die DU Tiere die signifikant höheren Werte für Fett- und Muskelfläche auf. Im Rassenvergleich zeichnen sich die SF Tiere mit relativ hohen Werten für die Muskelfläche aus. Während sich für die Marmorierung zwischen den Rassen keine Unterschiede ergaben, erreichte die SF Weidegruppe gegenüber der SF Stallgruppe einen signifikant höheren Wert. Bezüglich der L-Werte werden die signifikant besseren Werte bei den DU Stallgruppen gegenüber den Weidegruppen erzielt, während umgekehrt bei SF die Weidegruppe die besseren Werte aufweist. Die L* Werte zeigten Abhängigkeiten von der Haltungsform wenngleich bei den Rassen unterschiedlich. Hinsichtlich des Dripverlustes gab es zwischen den Haltungsformen tendenziell bessere Werte bei der Weidehaltung wobei die Werte für SF ein geringeres Wasserbindevermögen ausweisen. Bezüglich der $\mathrm{pH}$ Werte ergaben sich zwischen den Rassen signifikante und nicht gerichtete haltungsabhängige Unterschiede bei SF und DS, währen auch hier die DU Weidegruppe bessere Werte erreichte. 
Tabelle 3

Fleischqualitätsparameter der Populationen bei unterschiedlicher Haltungsform (Meat quality of different populations and housing systems)

\begin{tabular}{|c|c|c|c|c|c|c|c|c|c|}
\hline \multirow[t]{2}{*}{ Rasse } & \multicolumn{3}{|c|}{ Stall } & \multirow{2}{*}{$\begin{array}{c}\text { Weide } \\
\mathrm{LSM} \pm \mathrm{SD}\end{array}$} & \multirow[b]{2}{*}{ Stall } & \multicolumn{4}{|c|}{ Signifikanz } \\
\hline & & & & & & Weide & & Stall & Weide \\
\hline & & $\underset{+)}{\text { Intramuskul. Fett }}$ & $\%$ & & & & & 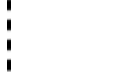 & \\
\hline SF & 21 & $1,71 \pm 0,46$ & & $1,63 \pm 0,43$ & & & n.s. & B & B \\
\hline DS & 10 & - & & $1,54 \pm 0,50$ & & & - & - & B \\
\hline $\mathrm{Du}$ & 19 & $\begin{array}{c}2,47 \pm 0,53 \\
\text { Fettfläche }^{+)}\end{array}$ & $\mathrm{mm}^{2}$ & $2,30 \pm 0,49$ & & & n.s. & A & A \\
\hline SF & 21 & $22,6 \pm 3,7$ & & $23,9 \pm 3,7$ & & & n.s. & - & A \\
\hline DS & 19 & $28,5 \pm 3,7$ & & $25,8 \pm 3,5$ & & & n.s. & B & A \\
\hline $\mathrm{Du}$ & 19 & $\begin{array}{c}\text { 19,5 } \pm 3,7 \\
\text { Muskelfläche }^{+)}\end{array}$ & $\mathrm{mm}^{2}$ & $12,8 \pm 3,5$ & $\mathrm{a}$ & b & $*$ & A & B \\
\hline SF & 21 & $43,5 \pm 3,5$ & & $42,7 \pm 3,3$ & & & n.s. & A & A \\
\hline DS & 19 & $32,6 \pm 2,3$ & & $34,5 \pm 2,2$ & & & n.s. & B & B \\
\hline $\mathrm{Du}$ & 19 & $\begin{array}{c}\text { 42,8 } \pm 2,5 \\
\text { Marmorierung }\end{array}$ & Pkt & $36,2 \pm 3,4$ & $\mathrm{a}$ & b & $* * *$ & A & B \\
\hline SF & 21 & $1,60 \pm 0,55$ & & $2,36 \pm 0,52$ & b & $\mathrm{a}$ & $*$ & - &.- \\
\hline DS & 19 & $1,44 \pm 0,41$ & & $1,80 \pm 0,40$ & & & n.s. & - & - \\
\hline $\mathrm{Du}$ & 19 & $\begin{array}{c}2,11 \pm 0,54 \\
\mathbf{L} \text { (Farbhelligkeit) }^{+}\end{array}$ & & $1,90 \pm 0,51$ & & & n.s. & 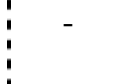 & - \\
\hline SF & 21 & $50,5 \pm 2,4$ & & $45,9 \pm 2,2$ & a & b & $*$ & A & - \\
\hline DS & 10 & - & & $47,9 \pm 2,3$ & & & - & - & - \\
\hline $\mathrm{Du}$ & 19 & $\begin{array}{c}45,6 \pm 1,2 \\
\mathbf{a}(\text { Rotanteil) }\end{array}$ & & $48,1 \pm 1,1$ & b & $\mathrm{a}$ & $* *$ & B & - \\
\hline $\mathrm{SF}$ & 21 & $9,09 \pm 0,87$ & & $8,23 \pm 0,83$ & & & n.s. & A & A \\
\hline DS & 10 & - & & $7,27 \pm 0,70$ & & & - & - & B \\
\hline $\mathrm{Du}$ & 19 & $\begin{array}{c}7,37 \pm 0,64 \\
\text { b (Gelbfärbung) }\end{array}$ & & $7,57 \pm 0,60$ & & & n.s. & B & B \\
\hline SF & 21 & $4,71 \pm 0,84$ & & $3,51 \pm 0,80$ & $\mathrm{a}$ & b & $*$ & A & - \\
\hline DS & 10 & - & & $3,10 \pm 0,85$ & & & - & - & - \\
\hline $\mathrm{Du}$ & 19 & $\begin{array}{c}2,60 \pm 0,43 \\
\text { Dripverlust }^{+}\end{array}$ & $\%$ & $3,72 \pm 0,42$ & b & a & $* * *$ & B & - \\
\hline SF & 21 & $7,05 \pm 0,90$ & & $5,92 \pm 0,86$ & & & n.s. & A & A \\
\hline DS & 10 & - & & $5,68 \pm 0,90$ & & & - & - & A \\
\hline $\mathrm{Du}$ & 19 & $\begin{array}{c}\text { 4,59 } \pm 1,20 \\
\text { pH45 Schinken }\end{array}$ & & $3,99 \pm 1,10$ & & & n.s. & B & B \\
\hline SF & 21 & $5,74 \pm 0,19$ & & $5,96 \pm 0,18$ & & & n.s. & B & B \\
\hline DS & 19 & $5,87 \pm 0,16$ & & $6,41 \pm 0,15$ & b & $\mathrm{a}$ & $* * *$ & B & A \\
\hline $\mathrm{Du}$ & 19 & $\begin{array}{c}\text { 6,35 } \pm 0,18 \\
\text { pH45 Kotelett }\end{array}$ & & $5,95 \pm 0,17$ & a & b & $* *$ & A & B \\
\hline SF & 21 & $5,38 \pm 0,04$ & & $5,43 \pm 0,04$ & b & $\mathrm{a}$ & $*$ & $\mathrm{C}$ & $\mathrm{C}$ \\
\hline DS & 19 & $6,08 \pm 0,19$ & & $6,53 \pm 0,18$ & b & $\mathrm{a}$ & $* *$ & B & A \\
\hline $\mathrm{Du}$ & 19 & $6,38 \pm 0,16$ & & $6,24 \pm 0,21$ & & & n.s. & A & B \\
\hline
\end{tabular}

unterschiedlich kleine Buchstaben = Signifikanz zwischen den Haltungsformen, innerhalb einer Rasse, ${ }^{*} \mathrm{P} \leq 0,05,{ }^{* *} \mathrm{P} \leq 0,01,{ }^{* * *} \mathrm{P} \leq 0,001$ unterschiedlich große Buchstaben $=$ Signifikanz zwischen den Rassen, innerhalb der Haltungsform, , ${ }^{*} \mathrm{P} \leq 0,05$,

$+)$ M.l.d.

4. Diskussion

Erwartungsgemäß zeigten fast alle Gruppen auf Grund des Futterangebotes relativ niedrige Mastleistungen, die extensiveren Haltungsbedingungen entsprechen. Auch die ad libitum Grasfutteraufnahme konnte dies nicht ausgleichen. Über diese tendentiell niedrigeren Zuwachsraten in der Freilandhaltung liegen zahlreiche Literaturinformationen vor (SCHNEIDER und WAGNER, 1996; SPITSCHAK, 1997; MICKLICH und 
MATTHES, 1999) Als Grund für geringere Zuwachsraten werden bei Weidehaltung jahreszeitlich wechselndes Futterangebot, in den warmen Sommermonaten geringere Fresslust oder Parasitenbefall mit daraus reduziertem Futterverzehr angesehen (DUFFEY, 1995; EIDGEN. FORSCHUNGSANST., 1996) Aus anderen Arbeiten ist die Zusammensetzung des Mageninhaltes von Schwarzwild bekannt. Sie zeigen, dass der Anteil pflanzlicher Nahrung aus Kräutern und Gräsern 80 bis 90\% betrug, der Rest tierischen Ursprungs war und nur ein relativ geringes Energieniveau erreicht (OLOFF, 1951; JANDA, 1958; HECK und RASCHKE, 1980; MEYNHARDT, 1986). Die Qualität des angebotenen Weidefutters im vorliegenden Versuch entsprach mittleren Werten. Monatliche Futterproben des Weidefutters ergaben Energiegehalte von 8,7 bis 9,3 MJ/kg TS was einer gewichteten Futterwertzahl nach KLAPP und BOBERFELD (1988) von 5,2 entspricht (MICKLICH, 1996). HANSSON (1991) untersuchte den Einfluss der Bewegung während der Mast indem die Tiere 5 mal wöchentlich eine Strecke von 500 Metern laufen mussten, konnte aber keinen Einfluss auf die Mastoder Schlachtleistungen beschreiben. Das gilt auch für die vorliegenden Untersuchungen in welchen keine gerichteten Einflüsse in Abhängigkeit von der Haltungsform nachgewiesen werden konnten.

Bezüglich der Fleischqualitätsmerkmale zeigt die Tabelle 3 nur in wenigen Merkmalen signifikante Unterschiede in Abhängigkeit von der Haltungsform, jedoch weit mehr Unterschiede zwischen den Populationen. Beim intramuskulären Fettgehalt wird auf Grund der geschmacksbildenden (MEYER und BARBE, 2000) und ernährungswirtschaftlichen (ENDER et al., 1997) Komponenten ein Optimum von 2\% angestrebt (CMA, 1998). Diesen Wert erreichen lediglich die DU Gruppen unabhängig von der Haltungsform. Die Rasseabhängigkeit bestätigt sich auch durch den Umstand, dass diese relativ hohen Werte bei DU sowohl bei niedrigeren als auch höheren Tageszunahmen erreicht werden. Das gilt auch für das Verhältnis der Fett- zur Fleischfläche, welches bei der Rasse DU in beiden Haltungsformen erheblich günstiger ausfällt, nämlich 1:2,83 bzw 1:2,88. Bezüglich der Marmorierung zeigt die SF Gruppe der Weidehaltung die bessere Punktzahl zugunsten der Weidegruppe. In allen diesen wertbestimmenden Merkmalen erreichen die DS die ungünstigsten Werte. Das Verhältnis der Fettflächen zu den Fleischflächen betrug zum Beispiel bei dieser Population in den beiden Haltungsformen 1:1,14 bzw. 1:1,34. Dieses Ergebnis stimmt mit Aussagen von KUHN et al. (1997) bezüglich der Fleischqualität des DS überein. Wenn auch bei Weidehaltung, außer bei Du, nur tendenziell verringernde Effekte bei den Fettmerkmalen (intramuskuläres Fett, Fettfläche) nachgewiesen werden konnten, so kann bei dieser Haltungsform mit einer Erhöhung der n-3 gegenüber den n-6 Fettsäuren gerechnet werden (DUFEY, 1992; ENDER et al., 1997). Über diese positiven Wirkungen der Weidehaltung auf die Erhöhung der n-3 Fettsäuren bei Schweinen und anderen Tierarten berichten auch andere Autoren (ENSER und WOOD, 1995; KRACHT et al., 1996; LEIBETSEDER et al., 1996; ENDER et al., 1997). Die Ergebnisse der Chromometer L*-Werte für die Farbhelligkeit ebenso wie die Werte für die Gelbfärbung zeigen rassenabhängig signifikante jedoch nicht gerichtete Einflüsse der Haltungsform und Unterschiede zwischen den Gruppen SF und DU. Der L*-Wert der SF Stallgruppe liegt mit 50,5 nahe dem Grenzbereich für PSE (<50 HONICKEL, 1998 bzw. $<51$ REICHARDT et al., 2001), nach CMA Prüfsiegel (1998) jedoch immer noch unter $<53$ als zugelassenem Wert. Diese höheren Werte der SF Stallgruppe deuten jedoch auf eine mangelhafte Fleischqualität hin, zumal wenn sie nach FIEDLER et al. (2002) 
mit höheren Dripverlusten verbunden sind. Signifikant besser mit $L^{*}=42,7$ zeigt sich die Weidegruppe dieser Population. Die DU Weidegruppe erreicht den absolut niedrigsten Wert. Hinsichtlich des Wasserbindevermögens werden für die Weidegruppen tendentiell geringere Dripverluste festgestellt, was bei diesem wichtigen Qualitätskriterium möglicherweise auf den Bewegungseinfluss zurückzuführen ist. Bezüglich des pH Wertes werden in den Prüfplänen der CMA (1998) für Schweinefleisch Werte von $<6,0$ gefordert. Zwischen den Rassen bestanden zwar überwiegend signifikante Unterschiede, zwischen den Haltungsformen waren diese vorhanden, jedoch nicht gerichtet. Gleiches berichten auch ADGE und EIDAM (1989).

Insgesamt ist festzustellen, dass bezüglich der Fleischqualität bei extensiver GetreideGrünfuttermast der Schweine mit Weidehaltung, kaum Unterschiede zwischen im Stall oder auf der Weide gehaltenen Tieren festgestellt werden konnten. Ausnahmen bilden die bei einzelnen Populationen beobachteten besseren Werte in der Farbhelligkeit oder im Wasserbindevermögen. Gleiche Aussagen treffen VAN DER VAL (1991) und HÖGES (1998) aus Beobachtungen mit Scharrelschweinen. Neben der diskutierten Fettsäureproblematik liegen Vorteile dieser Mastform möglicherweise in der Verbraucherakzeptanz, so dass ein ökonomischer Effekt bei Direktvermarktung unter dem Gesichtspunkt von ökologisch erzeugtem Fleisch für einen begrenzten Markt erzielbar ist.

\section{Literatur}

ADGE, K; EIDAM, K.H.:

Erste Untersuchungen über den Einfluss extensiver Haltung und Fütterung auf die Mastleistung und Schlachtkörperqualität von Schweinen. Schweinezucht und Schweinemast 38 (1998), 42-44

CMA:

Deutsches Qualitätsfleisch aus kontrollierter Aufzucht. Prüfsiegel Programm „Schweinefleisch“, Prüfpläne (1998)

DUFEY, P.A.:

Vergleich unterschiedlicher Mastformen bei Schweinen. Teil 2: Fleischqualität. Landwirtschaft Schweiz 5 (1992), 581-586

DUFEY, P.A.

Fleisch- und Fettqualität bei Schweinemast mit Weidegang. Agrarforschung 2 (1995), 153-156

EIDGENÖSSISCHE FORSCHUNGSANST. f. VIEHWIRTSCHAFTLICHE PRODUKTION Landwirtschaft im Brennpunkt - wir forschen für eine gute Fleischqualität (1995)

ENDER, K.; NÜRNBERG, K.; PAPSTEIN, H. J.:

Die zwei Seiten des Fettes - eine Standortbestimmung. Arch. Tierz., Dummerstorf 40 (1997) 1, 35-45

ENSER, M.; WOOD, J.D.:

Effect of diet on intramuscular n-6 and n-3 polyunsaturated fatty acids in cattle, sheep and pigs. Proc. $2^{\text {nd }}$ Dummerstorf Muscle-Workshop „Muscle growth and meat quality“ Rostock 17-19 $9^{\text {th }}$ May (1995), 125-132

FIEDLER, I.; KUHN, G.; HARTUNG, M.; KÜCHENMEISTER, U.; NÜRNBERG, K.; REHFELD, CH.; HUBER, K.; KLOSOWSKA, D.:

Auswirkungen des Malignen Hyperthermie-Syndroms (MHS) auf Fleischqualität, Muskelfasereigenschaften und Stoffwechselkriterien des M. longissimus dorsi von Pietrain-Schweinen. Arch. Tierz., Dummerstorf 44 (2001) 2, 203-217

HANSSON, I.; LUNDSTRÖM, K.; ENFÄLT, A.C.; KARLSSON, A.; ESSEN-GUSTAVSSON, B.; HAKANSSON, J.:

Effect of moderate indoor exercise on carcass composition, mest quality and muscle encyme activities in pig. Proc. $37^{\text {th }}$ ICOMST (1991), 103-106

HECK, L.; RASCHKE, G.:

Die Wildsauen, Naturgeschichte - Ökologie - Hege und Jagd. Verlag Paul Parey, Hamburg, Berlin, 1980

HÖGES, J.L.:

Alternativen in der Schweinehaltung. Verlag Eugen Ulmer, Stuttgart, 1998 
HONIKEL, K.O.:

Physikalische Messmethoden zur Erfassung der Fleischqualität. In: BRANSCHEID, W. et al.: Qualität JANDA, M.: von Fleisch und Fleischwaren. Bd. 2, Deutscher Fachverlag, Frankfurt/M, 1998

Die Nahrung des Schwarzwildes - sus srofa L. im Mittelgebirgsgebiet von Stiavnica. Säugetierkundliche Mitteilungen 6 (1958), 67-74

KLAPP, E.; BOBERFELD, W.:

Kräuterbestimmungsschlüssel für die häufigsten Grünland- und Rasengräser. Verlag Paul Parey, Berlin, Hamburg, 2. Auflage (1988)

KRACHT, W.; JEROCH, H.; MATZKE, W.; NÜRNBERG, K.; ENDER, K.; SCHUMANN, W.:

The influence of feeding rape seed on growth and carcass fat quality of pigs. Fett/Lipid 98 (1996), 343351

KUHN, G.; HARTUNG, M.; FALKENBERG, H.; NÜRNBERG, G.; LANGHAMMER, M.; SCHWERIN, M.;

ENDER, K.:

Wachstum, Körperzusammensetzung und Fleischbeschaffenheit von im Fettansatz genetisch differenten Schweinen. Arch. Tierz., Dummerstorf 40 (1997) 4, 345-355

LEIBETSEDER, J.:

Beeinflussung tierischer Fette durch die Fütterung. Arch. Tierz., Dummerstorf 39 (1996) 4, 333-345

MEYER, E.; BARBE, C.:

MEYNHARDT, H.:

Geschmack wird schlecht bezahlt. Bauern Zeitung 11 (2000), 63

MICKLICH, D.

Schwarzwildreport, mein Leben unter Wildschweinen. Neumann Verlag, Radebeul, 1986

Die Wirkung der Weidehaltung verschiedener Schweinerassen auf die Sukzession von Flussauengrünland und den physiologischen Zustand der Sauen. Univ. Rostock, Diss., 1996

MICKLICH, D.; MATTHES, H.D.:

Die Weidehaltung von Sauen verschiedener Rassen auf Flussauenstandorten. Arch. Tierz., Dummerstorf

OLOFF, H.B.: 42 (1999) 2, 161-173

REICHARDT, W; MÜLLER, S.; LEITERER, M.:

Farbhelligkeit, Hämpigment- und Eisengehalt im Musculus longissimus dorsi bei Thüringer Schweineherkünften. Arch. Tierz., Dummerstorf 44 (2001) 2, 219-230

SAS INSTITUTE INC.:

SAS/Stat. User's Guide, Version 6, $4^{\text {th }}$ Edition (1990)

SCHNEIDER, P.; WALTER, J.:

Ethologische Untersuchungen von Mastschweinen auf Weiden. Arch. Tierz., Dummerstorf 39 (1996) 3, 299-307

SPITSCHAK, K.:

Fruchtbarkeits- und Aufzuchtleistungen von Sauen mit Ferkeln in der Freilandhaltung. Arch. Tierz. 40 (1997) 2, 123-134

VAN der VAL, P. G.:

Free range pigs: carcass characteristics and meat quality. Proc. $37^{\text {th }}$ ICOMST (1991), 202-205

ZDS (Zentralverband der Deutschen Schweineproduktion):

Richtlinie für die Stationsprüfung auf Mastleistung, Schlachtkörperwert und Fleischbeschaffenheit beim Schwein (1997)

Eingegangen: 09.08.2000

Akzeptiert: 15.04.2002

Anschriften der Verfasser

Dr. agr. DELIA MICKLICH

Dorfstr. 17a

D-19406 Ruchow

Dipl. agr. Ing. HORST MÖHRING

Landschaftspflege GmbH

Am Bahndamm

D-19309 Lenzen

Prof. Dr. habil. agr. HEIDE-DÖRTE MATTHES,

Dr. agr. MARGITTA HARTUNG

Institut für die Biologie landwirtschaftlicher Nutztiere (FBN)

Wilhelm Stahl Allee 2

D-18196 Dummerstorf 